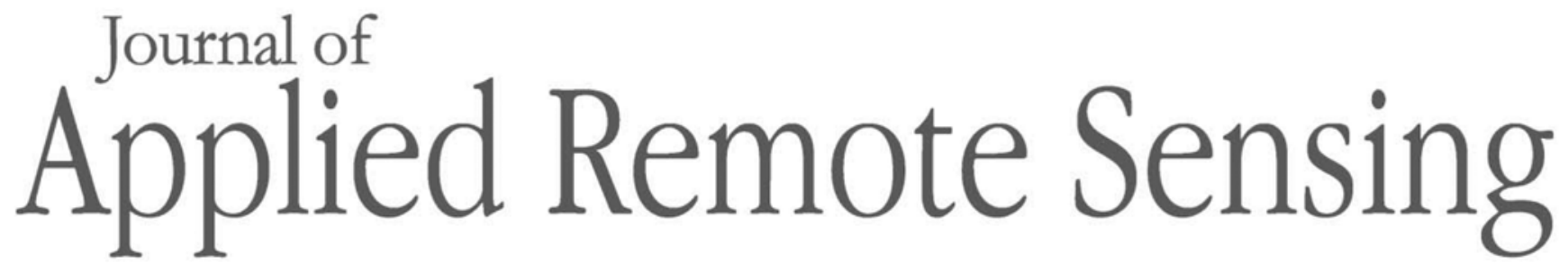

RemoteSensing.SPIEDigitalLibrary.org

\title{
Leveraging intercalibration techniques to support stray-light removal from Landsat 8 Thermal Infrared Sensor data
}

\author{
Aaron Gerace \\ Matthew Montanaro \\ Ryan Connal
}




\title{
Leveraging intercalibration techniques to support stray-light removal from Landsat 8 Thermal Infrared Sensor data
}

\author{
Aaron Gerace,* Matthew Montanaro, and Ryan Connal \\ Rochester Institute of Technology, Rochester, New York, United States
}

\begin{abstract}
Since its launch in 2013, the Thermal Infrared Sensor (TIRS) onboard Landsat 8 has exhibited artifacts in its image data that can be attributed to stray-light. A 3-year effort was initiated to develop a stray-light correction algorithm to support TIRS calibration. A methodology was developed to predict the additional (stray-light) signal on each detector from an estimate of the stray-light source locations in the sensor's out-of-field area. The initial version of the algorithm estimated the magnitude of out-of-field radiance sources through the use of geostationary wide-field thermal band imagers. However, this methodology necessitated a strong effort to cross calibrate the two sensors. Ultimately, a variation of the algorithm was implemented operationally into the United States Geological Survey ground system that utilizes image data from TIRS itself as an estimate of the out-of-field stray-light sources. This paper highlights the intercalibration techniques investigated while developing the stray-light correction algorithm. The impact of differing view-angles, spectral responses, and collection times on at-sensor radiance was considered to assess the feasibility of using data from Geostationary Operational Environmental Satellite geostationary instruments to estimate the out-of-field stray-light radiance incident on the TIRS detectors. Results of the studies presented here illustrate the complexities associated with intercalibration in the thermal and provide justification for the current form of the TIRS stray-light correction algorithm. (C) The Authors. Published by SPIE under a Creative Commons Attribution 3.0 Unported License. Distribution or reproduction of this work in whole or in part requires full attribution of the original publication, including its DOI. [DOI: 10.1117/1.JRS.12 .012007]
\end{abstract}

Keywords: intercalibration; cross calibration; thermal infrared; Landsat; Moderate Resolution Imaging Spectroradiometer; stray-light.

Paper 170495SS received Jun. 6, 2017; accepted for publication Sep. 21, 2017; published online Oct. 27, 2017.

\section{Introduction}

The radiometric intercalibration of space-based thermal infrared instruments is necessary to ensure that image data from two different sensors are consistent with each other when viewing the same location on the Earth. The importance of intercalibration became evident while developing the stray-light correction algorithm for the Landsat 8 Thermal Infrared Sensor (TIRS). To remove stray-light artifacts in imagery from the thermal instrument, a correction algorithm was constructed that relies on reference data from a different (but similar) thermal instrument to characterize the miscalibration of the TIRS instrument. However, the reference data must first be corrected to transform the image data from the reference sensor into image data that would have been acquired under the same viewing conditions and characteristics as the TIRS sensor. Specifically, the impact of differing relative spectral response (RSR) functions, sensor view-angles through the atmosphere, and image acquisition times on at-sensor radiance must be assessed and taken into account when comparing image data from the two sensors. The intercalibration methodology developed as part of the stray-light correction involved utilizing Terra/Moderate Resolution Imaging Spectroradiometer (MODIS) and Geostationary Operational Environmental Satellite (GOES)-N series thermal band data as reference data for TIRS.

*Address all correspondence to: Aaron Gerace, E-mail: gerace@ cis.rit.edu 
Although the cross calibration of these particular sensors is presented here as it pertains to the stray-light correction algorithm, the studies and methodologies highlight the scarcity of thermal reference data and illustrate the difficulty of intercalibration of thermal infrared instruments in general. This paper will provide an overview of the stray-light correction algorithm for the TIRS instrument, describe the reference data utilized by the algorithm, develop the methodology to cross calibrate the reference data, and compare the performance of the algorithm with and without properly cross calibrated reference data.

\section{Background}

The TIRS instrument on Landsat 8 provides continuity of thermal infrared data of the Earth for the Landsat program. The instrument is a push-broom architecture with a linear array of detectors for two spectral bands centered at 10.9 and $12.0 \mu \mathrm{m}$ (referred to as Landsat 8 band 10 and band 11, respectively). Image data from TIRS have exhibited significant variability that can be attributed to stray-light effects. These effects, which appear in the image data as nonuniform banding and seasonal variability in the absolute accuracy, have been well documented. ${ }^{1,2}$ A significant effort was initiated to better understand the origin of the stray-light and to investigate potential postprocessing methodologies that could be used to remove the stray-light image artifacts. Ultimately, a solution was achieved that relates knowledge of the stray-light sources in the TIRS out-of-field to additional signal on the TIRS focal plane, ${ }^{3,4}$ according to Eq. (1). In March 2017, the United States Geological Survey (USGS) began making corrected TIRS data available to users as part of the Landsat 8 standard radiance product.

$$
\text { TIRS_radiance }- \text { true_radiance }=a * \operatorname{sum}(\text { out-of-field radiances })+b .
$$

To briefly summarize the stray-light correction algorithm, the left-hand side of Eq. (1) represents the extra bias signal on a detector, where "TIRS_radiance" is the actual measured radiance on the detector and "true_radiance" is the radiance that should have been measured from the direct lineof-sight of the detector if the instrument did not have a stray-light problem. A calibrated reference dataset can be leveraged to determine true_radiance.

The right-hand side of Eq. (1) represents the stray-light radiance from the out-of-field. Reference data are required to provide radiance information of the out-of-field radiances and linear regression used to establish the $a$ and $b$ coefficients (see Refs. 3 and 4 for more details).

During the development of the TIRS stray-light correction algorithm, the scarcity of thermal reference data became apparent. As just described, reference data are required for two purposes in the stray-light correction process: (1) to quantify the additional recorded stray-light signal on each detector and (2) to quantify the incident out-of-field radiance. The ideal reference instrument(s) should have a similar spectral response, acquisition time, and view-angle through the atmosphere as the TIRS. Several instruments were considered to serve as reference data for the stray-light correction process, namely the Advanced Very High Resolution Radiometer (AVHRR), MODIS, GOES-N series instruments, and the TIRS itself.

The AVHRR instruments onboard MetOp A and B were initially considered as a potential solution to train the algorithm and serve as a source of out-of-field radiance. Bands 4 and 5 of AVHRR are spectrally similar to bands 10 and 11 of TIRS. However, the orbits of the satellites are such that when MetOp A (or B) has a similar ground track to Landsat 8, AVHRR's data are collected 45 min ahead of TIRS. Conversely, when MetOp A (or B) has near-coincident acquisition times with TIRS, its ground-track places the field-of-view of AVHRR outside that of Landsat 8/TIRS. Due to these inconsistencies in view-angle and/or collection times, the AVHRR sensor was excluded as a reference data source.

The Terra/MODIS sensor was initially dismissed as a source of reference data as its orbit places the field-of-view of TIRS at the extreme edge of the field-of-view of MODIS. However, for a short period after the launch as it was moved toward its operational orbit, Landsat 8 was maneuvered to under-fly Landsat 7 and (coincidentally) Terra/MODIS. From approximately 27 to 31 March 2013, Landsat 8/TIRS and Terra/MODIS collected data within 20 min of each other and through similar view-angles. Considering bands 31 and 32 of MODIS are spectrally similar to bands 10 and 11 of TIRS and that they are calibrated well within the $0.5 \%$ requirement, ${ }^{5}$ the 
data collected by Terra/MODIS during this time period were used as reference to quantify the additional stray-light radiance recorded by TIRS [see Eq. (1) (left)].

Once Landsat 8 achieved its operational orbit, Terra/MODIS data could no longer serve as a source of reference data (Landsat 8 is outside the field-of-view of MODIS at the equator and midlatitudes). An alternative source of thermal data to characterize the TIRS out-of-field was required. Geostationary instruments, such as the imagers on the GOES-N series (GOES-13, -14, and -15), offer a potential solution for the western hemisphere from the low to midlatitudes. However, these instruments contain only one band in the thermal (band 4) that is spectrally similar to TIRS band 10 and image the full disk only once every $3 \mathrm{~h}$. Despite these limitations, a geostationary solution to the stray-light correction process was pursued to develop methodologies that could be transferred to the GOES-R series once it became operational. GOES-R (now GOES16) was launched in November 2016 and its Advanced Baseline Imager (ABI) contains two bands that are spectrally similar to TIRS. It will image the entire disk every $15 \mathrm{~min}$ and significant effort is being conducted to calibrate the instrument, making it an attractive option for reference data.

Ultimately, a stray-light correction algorithm (described in Refs. 3 and 4) was implemented operationally into the USGS Landsat product generation system that uses Terra/MODIS and the TIRS data itself as reference. Terra/MODIS data obtained during the under-flight period were used for reference to characterize the true_radiance in Eq. (1). To characterize the out-of-field radiances in Eq. (1), the image data from the edge pixels of the TIRS interval itself were used as reference. Operationally, this method is attractive as it eliminates the need to perform intercalibration to compensate for differences in band shapes, collection times, and view-angles between two sensors. Furthermore, it offers a worldwide solution and avoids the need to ingest a secondary data source to support the stray-light removal. While a potential limitation of this method stems from the assumption that the edge pixels of a TIRS scene are representative of the out-offield, a solution was pursued that replaces TIRS data with GOES-N series data as reference.

The next section develops the methodology to cross calibrate thermal data from the GOES-N series sensors to utilize it as out-of-field reference data for the TIRS stray-light correction algorithm. The effects on at-sensor radiance due to spectral band shape, view-angle through the atmosphere, and collection times are characterized and applied to GOES image data.

\section{Methodology}

This section describes the techniques developed to support using geostationary thermal band image data in the stray-light correction algorithm. Specifically, a modeling effort and techniques designed to adjust at-sensor radiance to compensate for different RSRs and view-angles between two sensors are described. Additionally, a study is introduced that was designed to assess the impact of temporal acquisition offsets on the intercalibration process.

\subsection{Simulations Conducted}

To support the RSR and view-angle corrections suggested in this work, spectral at-sensor radiance was simulated using the moderate resolution atmospheric transmission (MODTRAN) code. ${ }^{6}$ Parameters that impact at-sensor radiance from 10 to $13 \mu \mathrm{m}$ were varied to generate

Table 1 Parameters and parameter variations used as input to MODTRAN.

\begin{tabular}{ll}
\hline \hline MODTRAN input parameters & \multicolumn{1}{c}{ Parameter variations } \\
\hline Surface temperature & 180 to $340 \mathrm{~K}(10 \mathrm{~K}$ increments $)$ \\
Emissivity & 12 variations of water, snow, trees, and desert \\
Atmospheric model & $\begin{array}{l}\text { Tropical, midlatitude summer, midlatitude winter, subarctic summer, } \\
\text { and subarctic winter }\end{array}$ \\
Water vapor scalar & $0.5,1.0,1.5$, and 2.0 \\
Detector view-angle & 0 deg to 60 deg (10 deg increments) \\
\hline \hline
\end{tabular}


a representative range of Earth's imaging conditions for spaceborne platforms. Specifically, referring to Table 1, the surface temperature input was varied in MODTRAN from $180 \mathrm{~K}$ to $340 \mathrm{~K}$ in increments of $10 \mathrm{~K}$. Twelve emissivities containing variations of water, snow, grass, trees, and desert were supplied as input to the model. Five default atmospheric profiles were used as input and a scale factor which adjusts their nominal water vapor content was varied to simulate different moisture levels in the atmosphere. Finally, the sensor-zenith angle was varied from $0 \mathrm{deg}$ (nadir) to $60 \mathrm{deg}$ in increments of $10 \mathrm{deg}$ to simulate a reasonable range of viewangles for wide field-of-view sensors. In all, 28,560 simulations were conducted to support the studies presented here.

\subsection{Relative Spectral Response Adjustment}

To utilize reference data from an alternative sensor, an adjustment must be made to the data to compensate for differences in the TIRS and the reference sensor's RSR functions. The RSRs of the sensors considered for the stray-light removal algorithm are shown Fig. 1. Note that bands 31 and 32 of MODIS and the 1120 and 1230 bands of the GOES-R/ABI sensor are spectrally similar to bands 10 and 11 of TIRS, making them attractive options for reference data. GOES-N band 4 is similar to band 10 of TIRS but not band 11 .

To develop a band-shape adjustment for the reference sensors considered in this work, the spectral at-sensor radiance generated from each MODTRAN simulation was spectrally sampled to all response functions shown in Fig. 1. The band-effective radiance for each TIRS band was

plotted against the band-effective radiance for the corresponding reference band and regression performed to find best-fit third-order polynomial functions to all data (see Fig. 2). The fit
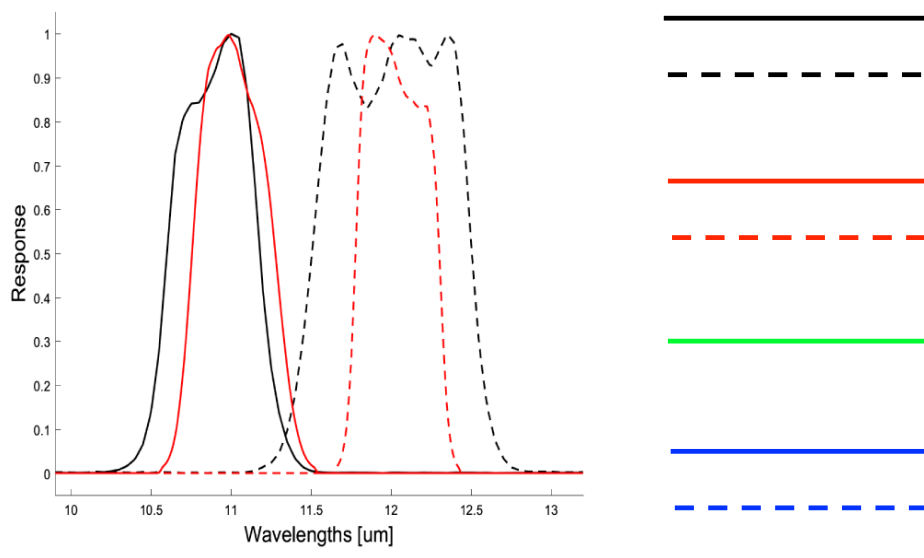

TIRS Band 10

TIRS Band 11

MODIS Band 31

MODIS Band 32

GOES Band 4

ABI Band 1120

ABI Band 1230
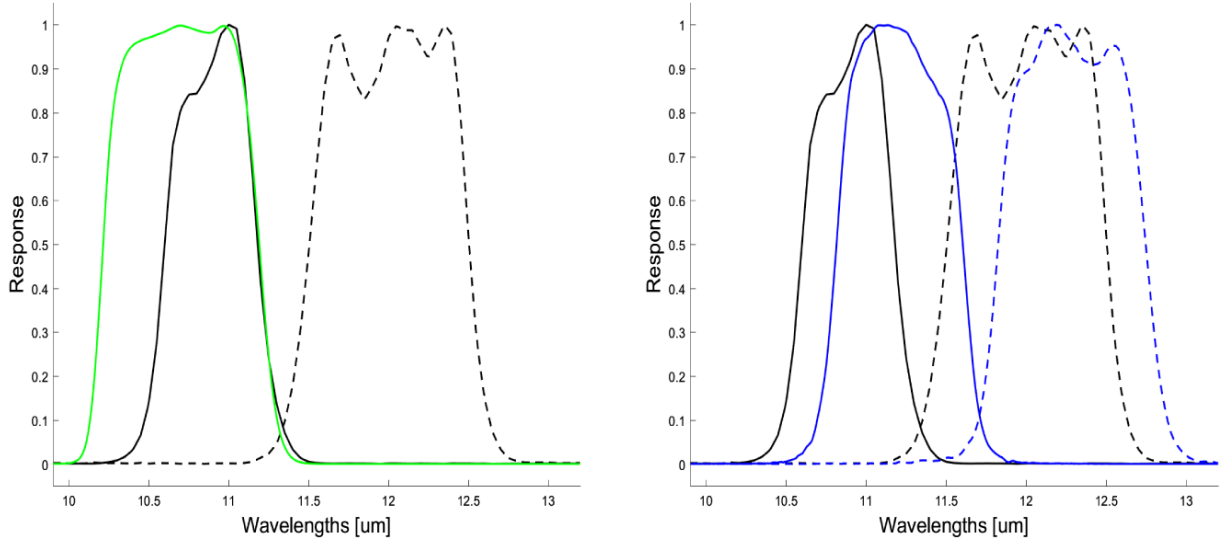

Fig. 1 The RSR functions of the reference sensors considered for the stray-light correction plotted against TIRS bands 10 and 11 . 

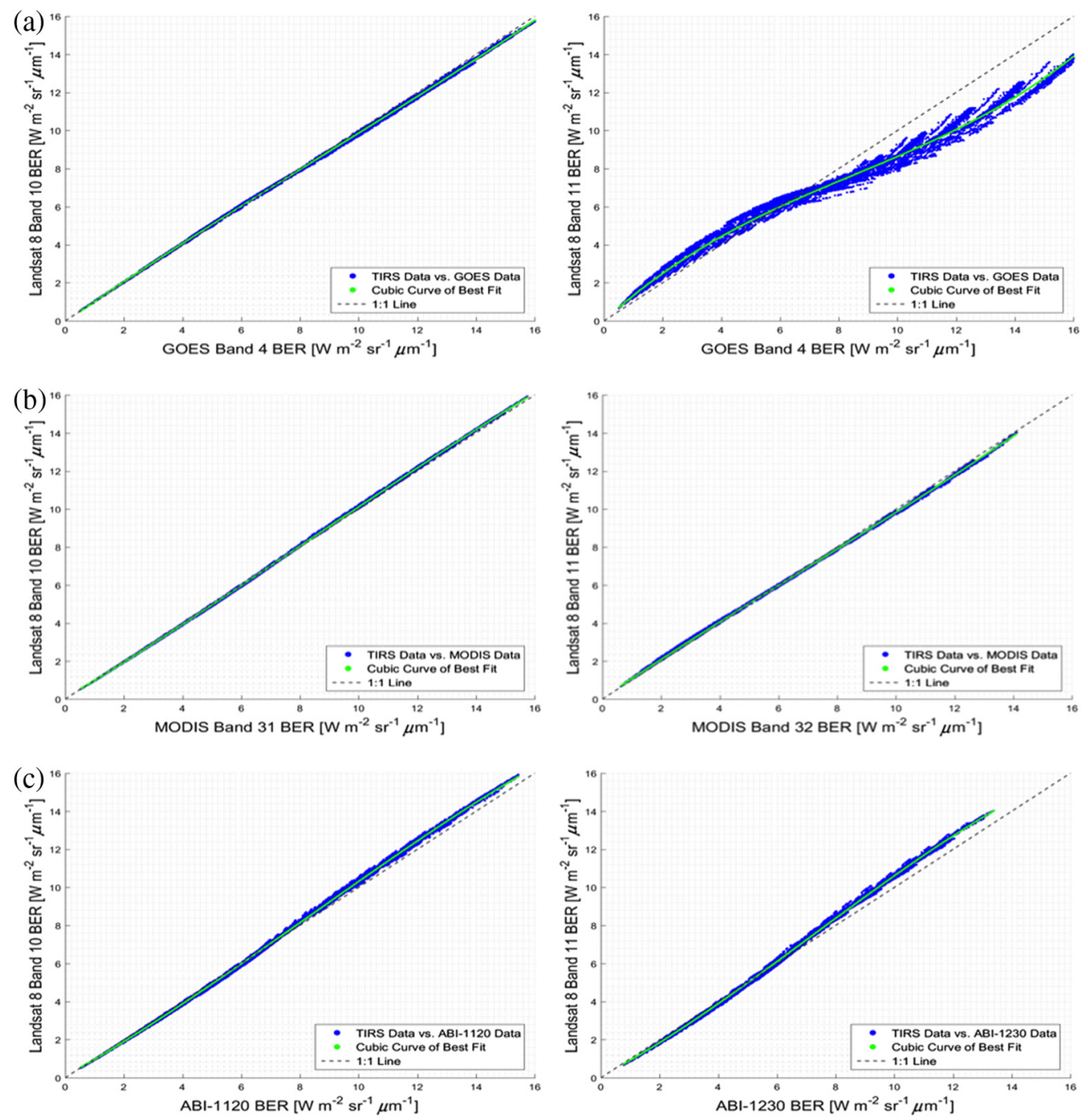

Fig. 2 MODTRAN-derived band-effective at-sensor radiances for TIRS versus a variety of thermal sensors: (a) GOES-N series, (b) MODIS, and (c) GOES-R series. The third-order polynomial fit functions provide a means to perform a band-shape adjustment from the sensor in question to the TIRS bands.

functions provide a means to transform the radiance data from the reference sensor into radiance data from the TIRS sensor.

To assess the impact of the band-shape adjustment on the intercalibration process, the root mean square (RMS) error between the best-fit curves and the data shown in Fig. 2 was determined and is reported in Table 2. As should be expected, due to their spectral similarities, performing a band-shape adjustment from MODIS to the corresponding TIRS bands introduces little variability. On the other hand, a band-shape adjustment from GOES band 4 to TIRS band 11 introduces, on average, nearly a 0.3 radiance unit error $(\sim 3 \mathrm{~K})$. Note that if a band-shape adjustment is not performed for GOES band 4 to TIRS band 11, an RMS error of 0.98 radiance units, on average, is introduced (or $\sim 10 \mathrm{~K}$ ).

\subsection{View-Angle Adjustment}

Although geostationary instruments typically have a small field-of-view due to their higher orbits, their effective view-angle through the atmosphere increases significantly as a function of distance from the subsatellite point on the Earth. To properly utilize thermal data collected from these platforms as reference, an adjustment should be made to account for their effective 
Gerace, Montanaro, and Connal: Leveraging intercalibration techniques to support stray-light...

Table 2 RMS errors $\left(\mathrm{W} / \mathrm{m}^{2} / \mathrm{sr} / \mu \mathrm{m}\right)$ between data and best-fit curves shown in Fig. 2 .

\begin{tabular}{lcc}
\hline \multicolumn{3}{c}{ Band effective radiance (RMSE) } \\
\hline Band & RMSE with Landsat 8/band 10 & RMSE with Landsat 8/band 11 \\
\hline MODIS band 31 & 0.03994 & - \\
MODIS band 32 & - & 0.04756 \\
GOES-15 band 4 & 0.05040 & 0.28165 \\
ABI 1120 & 0.08326 & - \\
ABI 1230 & - & 0.09716 \\
\hline \hline
\end{tabular}

view-angle. To illustrate the impact of view-angle on at-sensor radiance, the simulations described in Sec. 3.1 are utilized. Figure 3(a) shows the normalized band-effective radiance as a function of view-angle for GOES-N band 4 for all 28,560 simulations. For each simulation, the band-effective radiance is normalized by its nadir-viewing band-effective radiance to provide a scale factor to perform the view-angle adjustment. The adjustment would involve dividing the image value at each pixel by the appropriate scale factor for the pixel's atmospheric view-angle to transform the pixel data as if it were collected at nadir.

To assess the impact of each parameter (i.e., surface temperature, atmospheric model, water vapor, and material emissivity) on at-sensor radiance as view-angle changes, the data from Fig. 3(a) are parsed by each parameter for the extreme temperatures, $180 \mathrm{~K}$ (blue) and $340 \mathrm{~K}$ (red). Figure 3(b) shows the variability in at-sensor radiance as atmospheric model (i.e., atmospheric profile) changes, Fig. 3(c) shows the variability in at-sensor radiance as water vapor changes, and Fig. 3(d) shows the variability in at-sensor radiance as emissivity (i.e., surface cover type) changes. Notice, by comparing the parsed datasets to the full dataset, that the surface temperature causes the majority of the spread in the data. In other words, at-sensor radiance is most sensitive to changes in surface temperature when viewing off-nadir. This is followed by atmospheric model and water vapor. Finally, for the simulations conducted here, emissivity has the least impact on thermal band at-sensor radiance when viewing off-nadir.

Information contained in Fig. 3 can be leveraged to perform a view-angle adjustment. For example, if the sensor view-angle, surface temperature, atmospheric model (or profile), water vapor, and material emissivity parameters for the reference sensor image data are known or can be estimated, then the data in Fig. 3 can be parsed to determine the scale factor to perform the view-angle adjustment to nadir.

Typically, sensor view-angle is delivered as an ancillary product or can be derived for each pixel in an image. Several algorithms exist for single-band and multiband sensors that can be applied to produce surface temperature products from thermal imagery. ${ }^{7,8}$ Atmospheric profiles can be obtained over a scene of interest to characterize the atmosphere. Emissivity datasets can be obtained globally that provide an estimate of emissivity over the wavelengths of interest. ${ }^{9}$ All this information can potentially be used to parse the data in Fig. 3 and perform a rigorous viewangle correction. Details of the view-angle correction applied to the reference data used in this work are presented in Sec. 4.

\subsection{Temporal Offsets Between Acquisition of TIRS and Reference Data}

Considering the similar spectral responses of TIRS and MODIS, the under-flight period described in Sec. 2 represents an attractive window for MODIS to serve as reference data as Landsat 8 flew through the center of the field-of-view of Terra/MODIS with approximately a 20-min offset in image acquisition times. To properly choose scenes from the limited data available from the under-flight period, a quantitative assessment of how various materials thermally change over 20 min was desired. Due to its high thermal inertia and spatial uniformity, water likely represents the best target material for reference thermal image data. To quantify this, several years of cloud-free Landsat 7/ETM+ and near-coincident (approximately a $20 \mathrm{~min}$ 

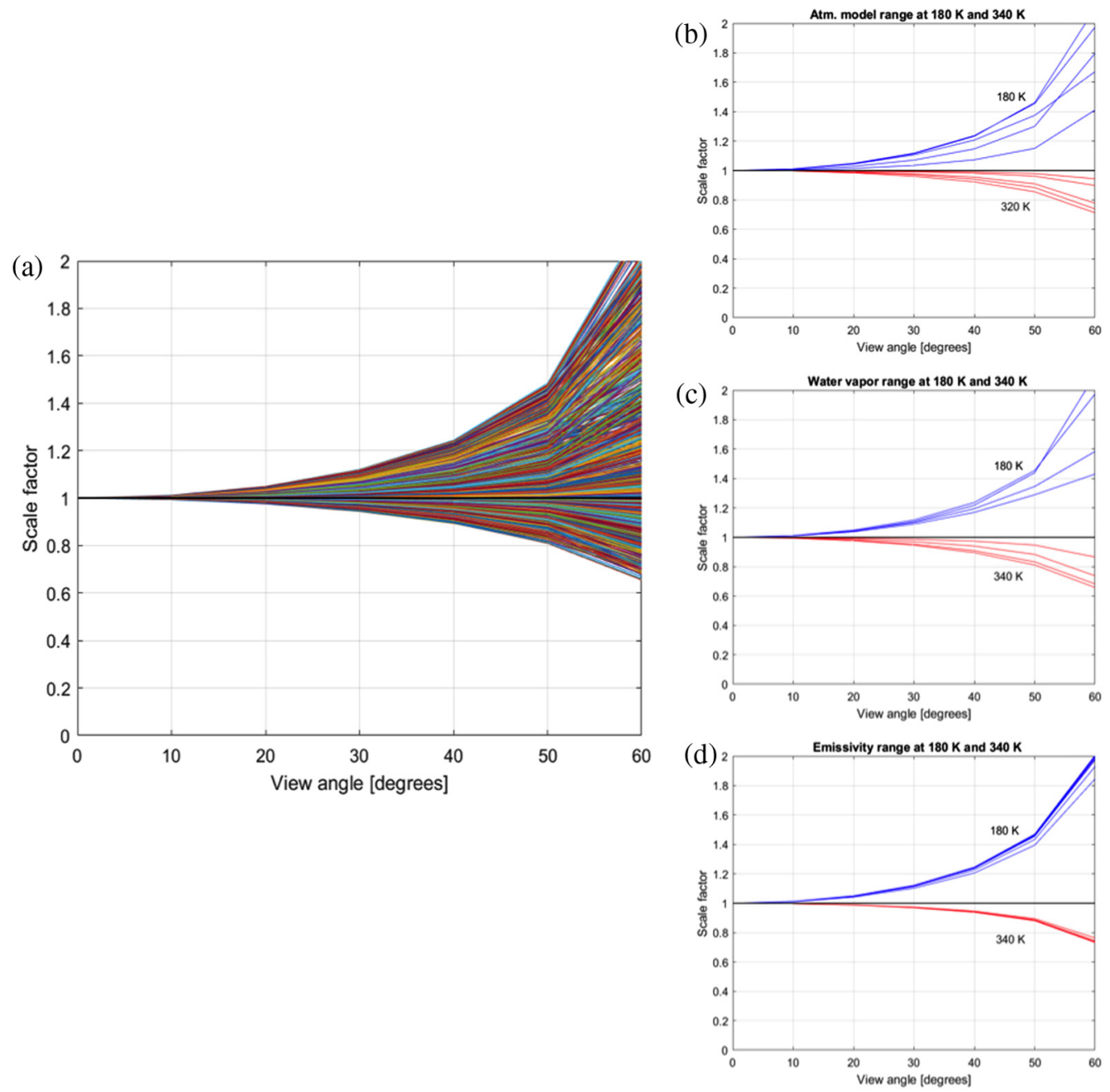

Fig. 3 Required view-angle adjustment scale factor for the GOES-N series imagers. The scale factor at a particular view-angle would be used to adjust the image data at that view-angle to a view-angle of nadir. (a) Scale factors for all modeled MODTRAN cases. To assess the sensitivity of each modeled parameter, the results were separated out by each parameter range at right. (b) Scale factors for the range of atmospheric models for the two extreme modeled surface temperatures (180 and $340 \mathrm{~K}$ ) and a single emissivity and water vapor value. (c) Scale factors for the range of water vapor values for the two surface temperatures and a single atmospheric model and emissivity. (d) Scale factors for the range of emissivities and a single atmospheric model and water vapor. The figures at right indicate that the surface temperature is the most sensitive parameter followed by atmospheric model, then water vapor, then emissivity.

collection offset) Terra/MODIS data were acquired over various materials (i.e., water, sand, snow, and forest). (Note that the limited availability of under-flight data precluded Landsat 8 from being used for this study.) Data were georegistered, converted to apparent temperature, and regions of interest (ROIs) obtained over uniform regions to obtain apparent temperature differences for the various materials. Figure 4 shows the difference in apparent temperature between ETM+ and MODIS as a function of average apparent temperature over the ROIs.

Note that (as expected) the smallest differences in apparent temperature were observed over water while the largest apparent temperature differences were observed over desert for the analysis conducted here. Although not comprehensive, this study illustrates the utility of using offshore water scenes as reference data for the stray-light correction algorithm.

With methodologies in place to reduce the impact of the key parameters (differences in RSRs, view-angle, and collection times) when cross calibrating reference data to TIRS, the training and validation of the stray-light correction algorithm could be performed. 


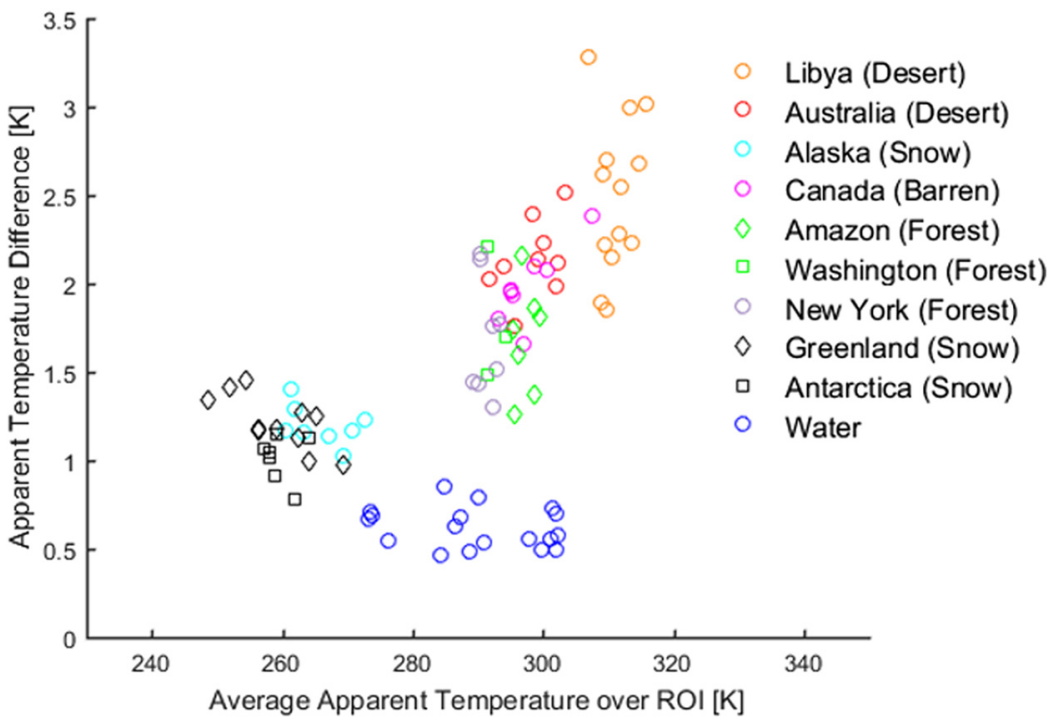

Fig. 4 Apparent temperature differences over various lands cover types between Terra/MODIS and Landsat 7/ETM+ image data (approximately a 20-min data acquisition offset).

\section{Stray-Light Correction Results and Discussion}

This section reports results of the TIRS stray-light correction algorithms in their current form. Specifically, MODIS and GOES-N series thermal reference data were obtained during the underflight period (described previously) and used to train the algorithm according to Eq. (1). To verify the fidelity of the procedures suggested in this paper, the MODIS reference data were compared to stray-light corrected TIRS data for four scenarios in which the out-of-field radiance was estimated from

1. TIRS data itself (known as "TIRS-on-TIRS"),

2. GOES-N series reference data without any adjustments,

3. GOES-N series reference data with only a band-shape adjustment, and

4. GOES-N series reference data with a band-shape and view-angle adjustment.

Comparison of images presented in Figs. 5-7 is generated by applying the appropriately trained stray-light correction algorithm to each dataset and then differencing the resulting data from MODIS reference data. All reference data were adjusted according to the discussions in Sec. 3 to train and verify scenarios 3 and 4 (described above) of the algorithm. For scenario 3, a band-shape adjustment was applied to the MODIS and GOES data to account for band-shape differences with TIRS (see Fig. 2). For scenario 4, considering at-sensor radiance is most sensitive to changes in surface temperature when viewing off-nadir, a split-window atmospheric correction algorithm ${ }^{7}$ was applied to the MODIS reference data to provide surface temperature information to support the view-angle correction. This step, although not appropriate for GOES-N band 4 sensors, was performed to illustrate the potential utility of the GOES-R ABI sensors, which have two thermal bands. All land pixels were masked out due to the discussion in Sec. 3.4 (i.e., significant heating of the land occurs over $20 \mathrm{~min}$ so MODIS land pixels should not be used as reference).

Figure 5 shows the TIRS-band 10/MODIS-band 31 difference images for the four scenarios described above for the Worldwide Reference System 2 (WRS2) scene path 220, rows 80 to 82 on March 31, 2013. The correction currently implemented operationally into the USGS ground system (denoted TIRS-on-TIRS) is shown in Fig. 5(b), followed by the GOES with no adjustment (c), the GOES with only a band-shape adjustment (d), and finally the GOES with the band shape and view-angle adjustments (e). Differences with MODIS (after a band-shape adjustment to the MODIS data) were calculated to highlight the differences between the TIRS product and a common reference dataset. There is significant banding in the uncorrected product [denoted Original in Fig. 5(a)] as evidenced in the nonuniformity of the water (which is expected to 


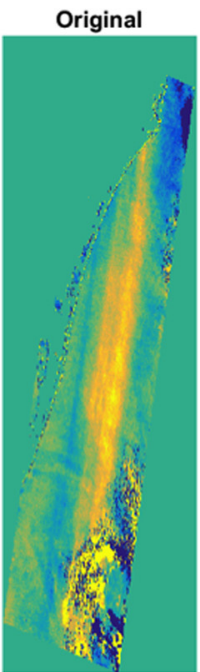

(a)

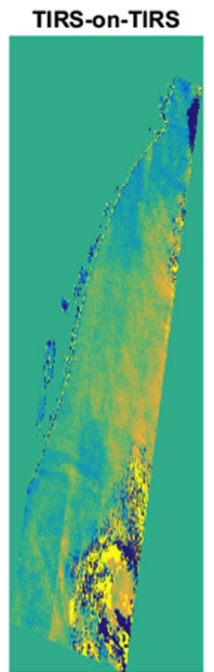

(b)

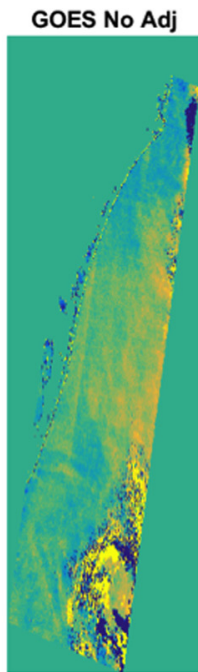

(c)

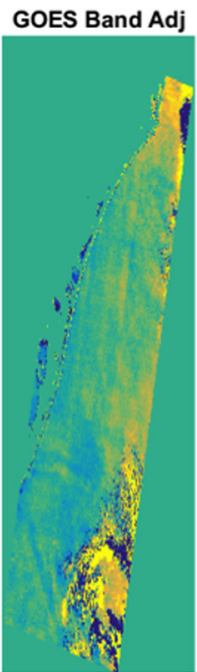

(d)

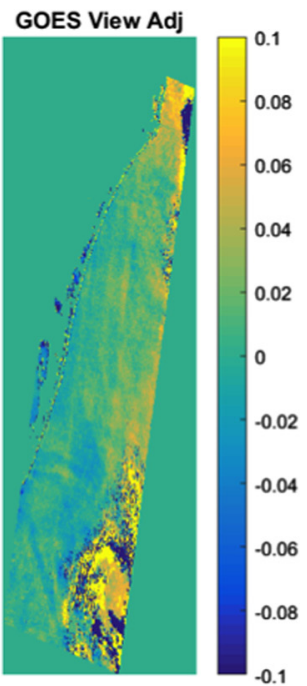

(e)

Fig. 5 Comparisons of Landsat 8/TIRS band 10 image products with various methods of stray-light correction for path 220, rows 80 to 82 on March 31, 2013. Each image represents the difference between the TIRS product and Terra/MODIS band 31 after a band-shape adjustment to MODIS. (a)-(e) The uncorrected product (original), the operational stray-light corrected product (TIRS-onTIRS), the stray-light corrected product using GOES-13 data with no adjustments (GOES No Adj), the stray-light corrected product using GOES-13 data with a band-shape adjustment (GOES Band Adj), and the stray-light corrected product using GOES-13 data with band-shape and view-angle adjustments (GOES View Adj). The contrast scale is in units of spectral radiance $\left(\mathrm{W} / \mathrm{m}^{2} / \mathrm{sr} / \mu \mathrm{m}\right)$.

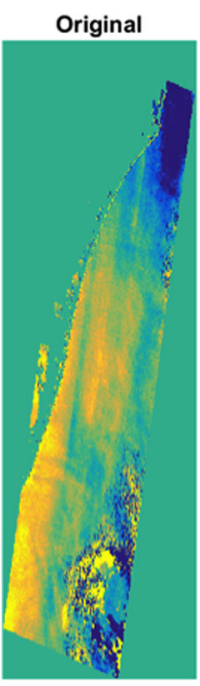

(a)

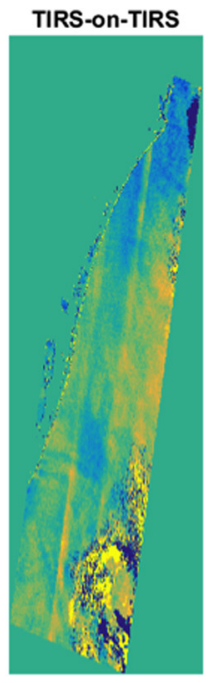

(b)

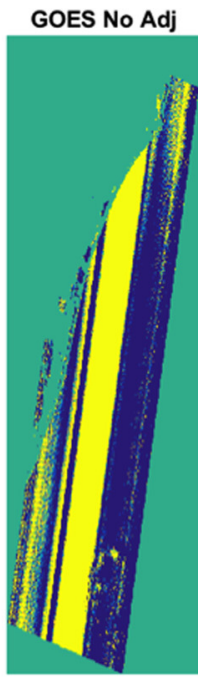

(c)

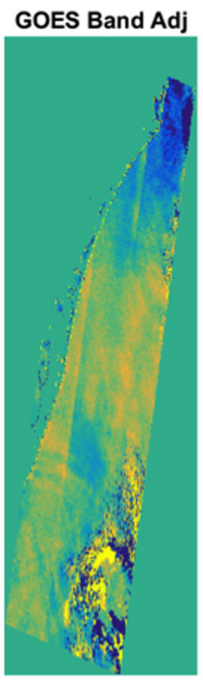

(d)

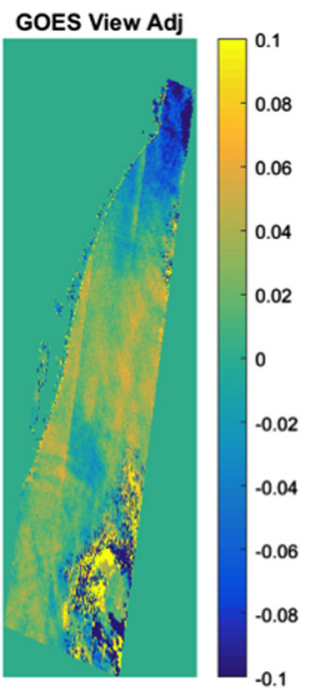

(e)

Fig. 6 Comparisons of Landsat 8/TIRS band 11 image products with various methods of straylight correction for path 220, rows 80 to 82 on March 31, 2013. Each image represents the difference between the TIRS product and the Terra/MODIS band 32 after a band-shape adjustment to MODIS. (a)-(e) The uncorrected product (original), the operational stray-light corrected product (TIRS-on-TIRS), the stray-light corrected product using GOES-13 data with no adjustments (GOES No Adj), the stray-light corrected product using GOES-13 data with a band-shape adjustment (GOES Band Adj), and the stray-light corrected product using GOES-13 data with bandshape and view-angle adjustments (GOES view adj). The contrast scale is in units of spectral radiance $\left(\mathrm{W} / \mathrm{m}^{2} / \mathrm{sr} / \mu \mathrm{m}\right)$. 
Path 006, Rows 70-71
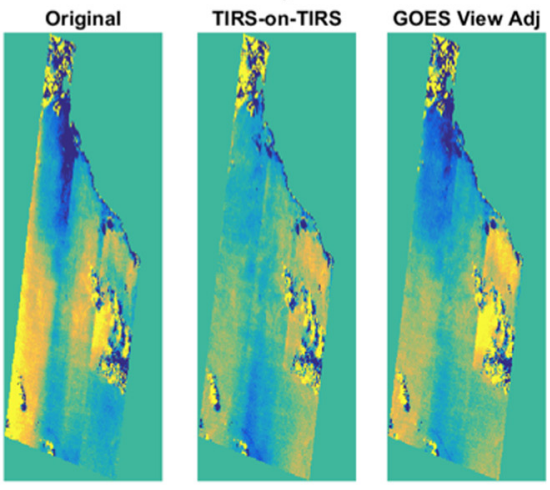

Path 013, Rows 32-33
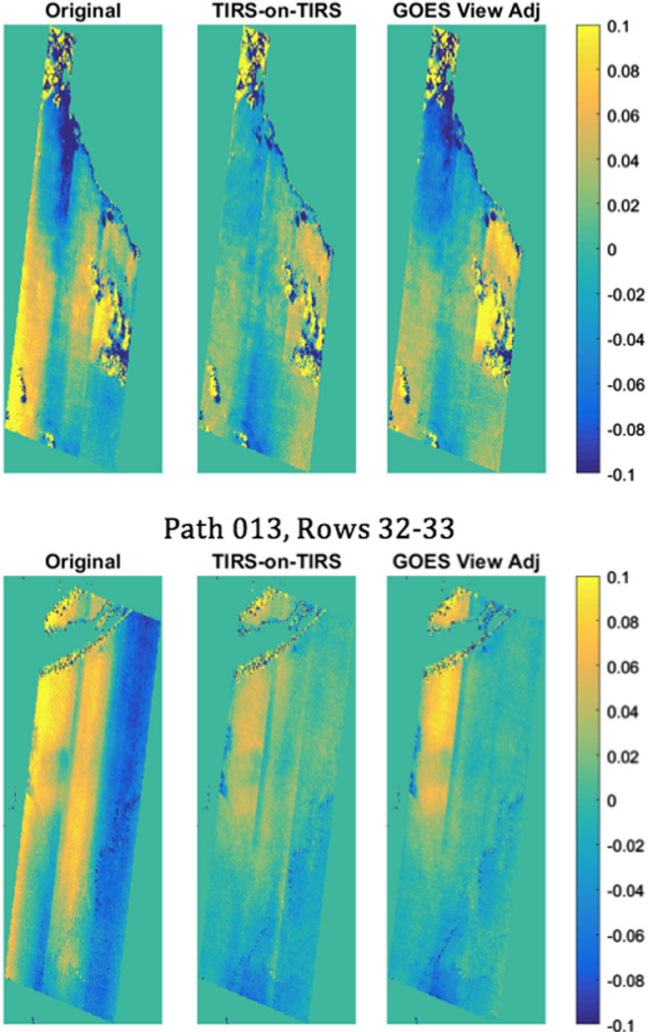

Path 016, Rows 37-40
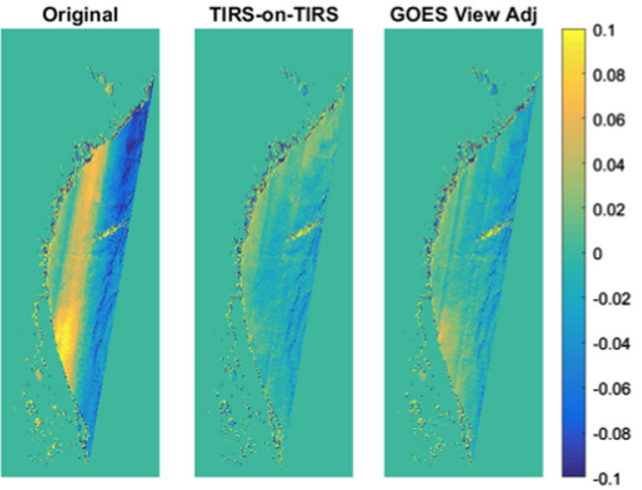

Path 022, Rows 27-31

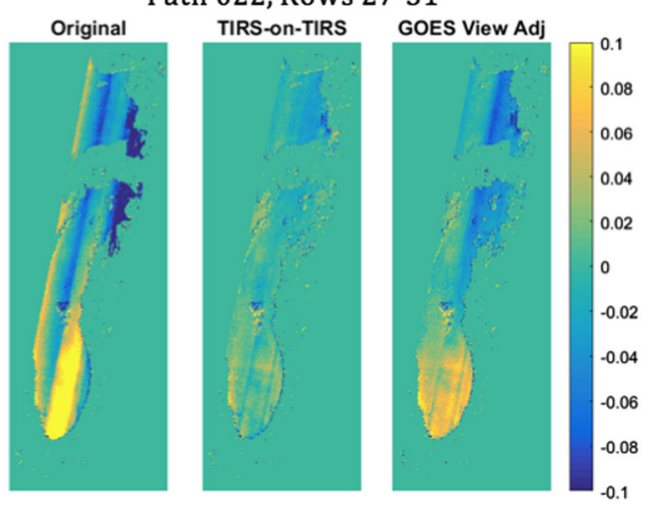

Fig. 7 Difference images for four WRS2 scenes between Terra/band 32 and Landsat 8/TIRS band 11 image products. The left image in each figure is the uncorrected product, the middle is the corrected product using TIRS data for the out-of-field radiance estimate (TIRS-on-TIRS), and the right image is the corrected product using adjusted GOES data for the out-of-field estimate.

be uniform). A considerable improvement in the banding is observed in the operational corrected product (TIRS-on-TIRS). Although not all banding is eliminated, the water in the scene is significantly more uniform over the uncorrected product. A similar result is observed for all the corrected products using GOES data. Considering the spectral shapes of TIRS band 10 and GOES band 4, which have significant overlaps [see Fig. 1(bottom left)], it is not surprising that the difference images associated with the "TIRS-on-TIRS" and "GOES No Adj" are quite similar. Therefore, applying the band-shape adjustment to the GOES out-of-field reference data has little impact on the fidelity of the algorithm results, as evidenced by visually comparing the "GOES No Adj" and the "GOES Band Adj" difference images. Applying the view-angle adjustment offers little improvement in the removal of stray-light for this case. Note that the effective view-angle of GOES was $\sim 46$ deg for this scene so more of an impact was expected from the view-angle correction for this scene. This observation is investigated later in this section.

Figure 6 shows the TIRS-band11/MODIS-band 32 difference images for the four scenarios and the original uncorrected product. In this case, by visually comparing the "TIRS-on-TIRS" difference image to the "GOES No Adj" and the "GOES Band Adj" difference images, the importance of the band-shape adjustment is apparent since GOES band 4 does not overlap spectrally with TIRS band 11. Again, applying the view-angle adjustment offered little improvement in the removal of stray-light for this case. In general, for both band 10 and band 11 (Figs. 5 and 6, respectively), the TIRS-on-TIRS and the GOES variations significantly outperform the uncorrected product.

Several additional scenes were corrected in the same manner as in Figs. 5 and 6 and the results from using adjusted GOES data for out-of-field radiance versus using TIRS data itself 
("TIRS-on-TIRS") are shown in Fig. 7. As previously mentioned, each image represents the difference between the TIRS band 11 product and the MODIS band 32 reference (note that only the original, TIRS-on-TIRS correction, and GOES with band and view-angle adjustment are shown in the figure). Overall, both corrections using the TIRS-on-TIRS and the GOES outof-field estimates provide a significant improvement in the scene uniformity over the uncorrected product. However, the TIRS-on-TIRS variation of the algorithm outperforms the adjusted GOES variation for the scenes presented here.

To determine a probable cause for this observation, a sensitivity analysis was performed to characterize the adjustments made to the GOES image data. Recall that since the surface temperature was found to be the most important factor influencing the at-sensor radiance for large viewangles, a scaling factor was derived for each view-angle using the MODIS-derived land-surface temperature products. That scaling factor was found by first selecting the surface temperature from the simulated cases from Sec. 3.1 and then taking the average scale factor over the remaining unknown variables. For example, considering the scene in Figs. 5 and 6, the average viewangle over the scene is $\sim 46 \mathrm{deg}$ and the estimated surface temperature is $\sim 280 \mathrm{~K}$. With this temperature estimate, the view-angle scaling factor was found by plotting all simulations from Sec. 3.1 with the estimated surface temperature (see Fig. 8). The black dotted line in the figure represents the average scaling factor at each view-angle when surface temperature is known. For the path 220 scenes with a view-angle of $46 \mathrm{deg}$, a view-angle scaling factor of 0.997 was applied, which is the value of the dotted line at $46 \mathrm{deg}$. However, as evident in the figure, there is a spread of about $\pm 2 \%$ of possible values at 46 deg due to the lack of knowledge of the atmosphere type, water vapor, and emissivity.

This residual error in view-angle adjustment, or error in out-of-field radiance, can be translated to an error in the stray-light correction via Eq. (1). To continue the scene example, the $\pm 2 \%$ error in the view-angle adjustment at $46 \mathrm{deg}$ would translate to errors in the stray-light corrected product of $1 \%$ to $3 \%$ in band 10 and $3 \%$ to $5 \%$ in band 11 .

This relationship illustrates the fundamental reason why utilizing data from a sensor other than TIRS will not necessarily result in a more accurate stray-light correction. To utilize data from another sensor as accurately as possible, highly accurate estimates of surface temperature, atmosphere type, water vapor, and emissivity should be known for high view-angles (larger than $\sim 30 \mathrm{deg}$ ). The TIRS-on-TIRS approach does not rely on external sensor data and, therefore, is not subject to the residual cross calibration errors. This fact, along with the availability of TIRS data worldwide (as opposed to ingesting data from numerous external sensors to obtain worldwide out-of-field radiance data), was the primary reason that the USGS implemented the TIRSon-TIRS approach operationally in the USGS Landsat ground system.

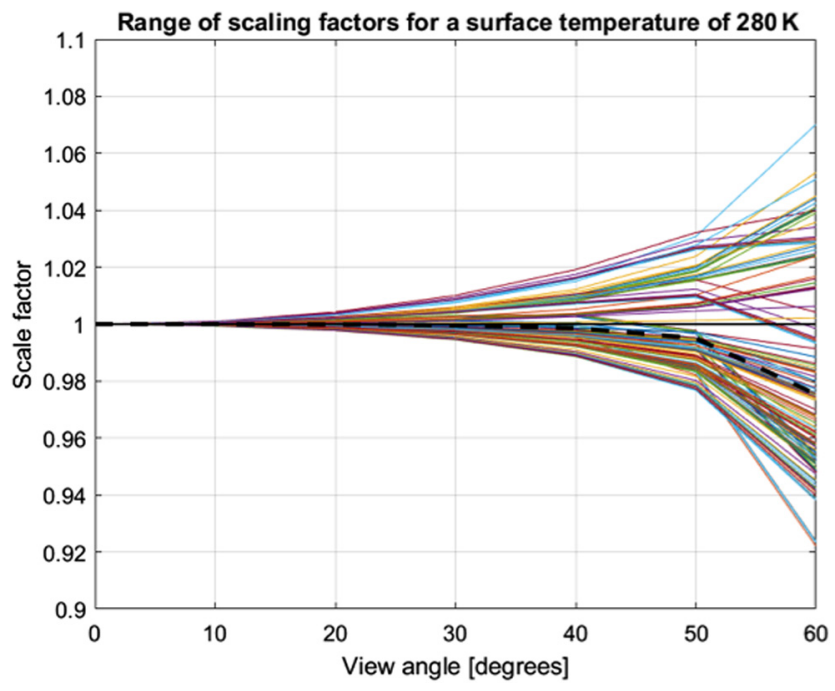

Fig. 8 View-angle adjustment scaling factors for the GOES-N series imagers given a surface temperature of $280 \mathrm{~K}$ based on the MODTRAN simulations in Sec. 3.1. The dotted black line represents the average scaling factor at each view-angle. 


\section{Summary}

The radiometric cross calibration of thermal band sensors is critical when comparing image data from one sensor to another. This fact became apparent while developing the stray-light correction algorithm for the Landsat 8 TIRS instrument. The algorithm required reference data to (1) calculate the magnitude of additional stray-light signal on each TIRS detector and (2) estimate the magnitude of the out-of-field stray-light sources for each TIRS scene. The algorithm proposed by Ref. 3 suggested utilizing wide-field image data from the GOES-N series imagers to estimate the out-of-field stray-light radiance for TIRS.

During the development of the methodology, it was discovered that several important factors influenced the cross calibration of the GOES thermal image data to the TIRS image data: RSR, view-angle through the atmosphere, and temporal offset in data acquisition. A band-shape adjustment was derived by running the MODTRAN code for a variety of surface temperatures, emissivities, atmosphere types, and column water vapors and spectrally sampling each top-ofatmosphere spectral radiance with the sensor's spectral response function. A regression between the band-effective radiances of the two sensors provides a means to scale image data from one sensor (GOES) into another sensor (TIRS).

Similarly, a view-angle adjustment can be derived from MODTRAN runs through various atmospheric view paths. An adjustment of image data from one sensor to another sensor due to view-angle differences can be performed if the view-angle, surface temperature, emissivity, atmosphere type, and water vapor are known. Analyses have demonstrated that these parameters must be well known to avoid introducing significant errors to the stray-light corrected product. For example, as presented in this work, a $2 \%$ error in the view-angle adjustment can result in an average error of $2 \%$ (band 10) to $4 \%$ (band 11) in the resulting stray-light corrected product.

Finally, the temporal offset between the two sensors' data acquisitions can be a large source of error. When viewing targets on the Earth, large water bodies exhibited the most stable atsensor radiance between data acquisitions due to the high thermal inertia versus other materials, such as sand and vegetation. The reference data used in the stray-light algorithm were obtained over large water bodies (oceans) within 20 min of TIRS data acquisition to minimize radiance differences due to natural heating effects.

The resulting corrected image products from TIRS using either the TIRS-on-TIRS approach or the adjusted GOES approach produced significantly improved results over the uncorrected product. The challenges of utilizing reference data from a sensor other than TIRS eventually led to the adoption by USGS of the TIRS-on-TIRS method for estimating the out-of-field stray-light radiance. Although the operational form of the correction algorithm is based on this TIRS-onTIRS approach, the methodology developed to utilize data from another sensor (GOES imagers) can be applied to any two similar sensors to cross calibrate their image data. As discussed here, the RSR, atmospheric view-angle, and proper Earth targets and data acquisition time offsets must be taken into account to use one sensor as reference for another.

\section{Acknowledgments}

The project described in this publication was supported by Cooperative Agreement No. G17AC00070 from the United States Geological Survey. Its contents are solely the responsibility of the authors and do not necessarily represent the opinions or policies of the USGS. Mention of trade names or commercial products does not constitute their endorsement by the USGS. This paper is submitted for publication with the understanding that the United States Government is authorized to reproduce and distribute reprints for governmental purposes.

\section{References}

1. A. Gerace and M. Montanaro, "Derivation and validation of the stray light correction algorithm for the thermal infrared sensor onboard Landsat 8," Remote Sens. Environ. 191, 246-257 (2017).

2. M. Montanaro, A. Gerace, and S. Rohrbach, "Toward an operational stray light correction for the Landsat 8 thermal infrared sensor," Appl. Opt. 54(13), 3963-3978 (2015). 
3. M. Montanaro et al., "Stray light artifacts in imagery from the Landsat 8 thermal infrared sensor," Remote Sens. 6(11), 10435-10456 (2014).

4. J. Barsi et al., "Landsat-8 thermal infrared sensor (TIRS) vicarious radiometric calibration," Remote Sens. 6(11), 11607-11626 (2014).

5. X. Xiong et al., "Terra and aqua MODIS thermal emissive bands on-orbit calibration and performance," IEEE Trans. Geosci. Remote Sens. 53(10), 5709-5721 (2015).

6. A. Berk, L. S. Bernstein, and D. C. Robertson, MODTRAN: A Moderate Resolution Model for LOWTRAN, (No. SSI-TR-124), Spectral Sciences Inc., Burlington, Massachusetts (1987).

7. Z. Wan, "New refinements and validation of the collection-6 MODIS land-surface temperature/emissivity product," Remote Sens. Environ. 140, 36-45 (2014).

8. J. C. Jiménez-Muñoz and J. A. Sobrino, "Error sources on the land surface temperature retrieved from thermal infrared single channel remote sensing data," Int. J. Remote Sens. 27(5), 999-1014 (2006).

9. G. C. Hulley and S. J. Hook, "The North American ASTER land surface emissivity database (NAALSED) version 2.0,” Remote Sens. Environ. 113, 1967-1975 (2009).

Aaron Gerace received his BS and MS degrees in mathematics from the College at Brockport in 2000 and 2002, respectively, and his PhD in imaging science from Rochester Institute of Technology in 2010. He is a senior researcher at Rochester Institute of Technology. His current research interests include calibration, intercalibration, and modeling. He is an active participant in the Landsat Calibration and Validation Group and has several journal articles on stray-light removal to support the calibration of TIRS on Landsat 8 .

Matthew Montanaro has been intimately involved in the calibration of the recent Landsat thermal band instruments. He supported the preflight calibration and postlaunch on-orbit characterization of the Landsat 8/TIRS. He codeveloped the stray-light correction algorithm that significantly reduces stray-light artifacts in TIRS imagery. He continues his Landsat thermal band calibration work as the Deputy Calibration Lead for the Landsat 9/TIRS-2 instrument.

Ryan Connal is pursuing his BS degree in imaging science at the Rochester Institute of Technology. He is a research assistant in the Digital Imaging and Remote Sensing Laboratory, where he supports the calibration of the TIRS instrument onboard Landsat 8. His current research seeks to leverage his background in calibration to assess the feasibility of deriving $100 \mathrm{~m}$ thermal inertia maps using readily available space-borne thermal data. 